\title{
Clubhouses as Essential Communities During the COVID-19 Pandemic
}

\author{
Liza Hinchey (1D) Amber Michon (1D) Jessica Drews • Megan Price • \\ Janay Christian $\cdot$ Francesca Pernice $\cdot$ Ralph Aquila
}

Received: 8 April 2021 / Accepted: 25 September 2021 / Published online: 27 October 2021

(C) The Author(s), under exclusive licence to Springer Nature India Private Limited 2021

\begin{abstract}
Intentional recovery communities, such as Clubhouses, exist as physical spaces for individuals living with Serious and Persistent Mental Illness. Due to the COVID-19 pandemic, it was necessary for these facilities to rapidly convert to virtual platforms. The aim of this study was to assess the extent to which virtual Clubhouse communities impacted the wellbeing of their members during the initial weeks of pandemic-related closures. Two hundred and eighty nine Clubhouse members across 19 countries responded to weekly measures of Clubhouse engagement, contact with other members, and well-being. A repeated measures multivariate analysis of covariance indicated that members with high levels of Clubhouse engagement reported higher mental and physical health ratings over time than those with low levels of engagement. These findings support the virtual Clubhouse model and highlight the efficacy of Clubhouses' rapid adaptations to the pandemic.
\end{abstract}

Keywords Clubhouse - COVID-19 pandemic . Engagement · Virtual communities · Well-being

L. Hinchey · A. Michon $(\square) \cdot$ J. Drews .

M. Price · J. Christian · F. Pernice

351 College of Education, Wayne State University,

Detroit, MI 48202, USA

e-mail: Amber.Michon@wayne.edu

R. Aquila

Fountain House, 425 W 47th St, New York, NY 10036, USA

\section{Introduction}

Clubhouse programs exist as physical spaces and places for people recovering from Serious and Persistent Mental Illness (SPMI) to participate as a community in daily activities that facilitate building social relationships, cultivating work skills, and sharing in an inclusive setting [3]. Located worldwide, Clubhouses serve as intentional communities composed of staff and adults living with SPMI who work together in a non-hierarchical environment to contribute to the functionality of the Clubhouse. The Clubhouse model, a form of psychosocial rehabilitation, operates by offering members opportunities to participate in activities such as culinary work, research, outreach, and running social programs within the Clubhouse. Additionally, these organizations emphasize a transitional model: by first becoming members of the Clubhouse community, individuals are able to eventually transition to greater participation in society at large [3]. A recent systematic review of evidence for this model found that Clubhouse engagement is associated with increased employment and quality of life, and fewer hospitalizations for those living with SPMI [7]. However, since the COVID-19 pandemic has reached a global scale of impact, many countries have implemented lockdowns, mandated social distancing restrictions, and closed businesses and services across various industries [1, 14]. Consequently, Clubhouse programs were among those many services 
shutting their doors and moving to a greater virtual and online platform.

While the physical risks of COVID-19 are clear [13], less well-articulated are the mental health concerns, this includes both mental health issues resulting from experiencing the pandemic conditions (e.g., fear, isolation) and heightened issues for those already experiencing struggles with mental health (for a current, broad literature review see Khalil 2020). The latter yields a vulnerable population documented with limited research articles and limited representation of countries [12]. Among the most universally noted mental health concerns during the pandemic are hopelessness, despair, grief, and bereavement [15], as well as loneliness and isolation [10]. However, new research has emerged suggesting that the mental health consequences have the potential to be more severe. In one of the earliest strongly impacted countries by COVID-19, Italy, over a third of a sample of Italian healthcare workers self-reported symptoms in the range of psychiatric morbidity as measured using the General Health Questionnaire (GHQ-12), which examines distress and hindrances to daily functioning as a means of identifying those likely to have or be at risk of psychiatric disorders [2]. Additionally, individuals living with psychiatric disorders have reported an increase in symptoms since the beginning of the pandemic, and the general public has experienced an overall worsening of mental wellbeing [16]. Particularly for people with SPMI, loneliness and isolation represent potential risk-factors for the worsening of psychiatric symptoms [9]—-thus, pandemic conditions may pose an even greater risk for these populations than for the general public.

Aware of the potential implications for mental health consequences of the pandemic, Clubhouses that support individuals with SPMI were required to respond in a novel way, particularly when stay at home orders were executed around the world. Shutdowns or "shelter in place" orders and physical or social distancing stipulations threatened access to their vital structured routines and community supports. Clubhouses, however, shifted their presence from one that was in-person to one that was virtual. These virtual Clubhouse communities extended wellness checks and resources to members and encouraged them to engage in new online platforms where they could connect with other members and complete activities parallel to those they would have done in their physical buildings. In order to better capture the activities of virtual Clubhouses, how their members were faring, and how they were continuing to support their members, Clubhouse International and partners facilitated distribution of surveys to obtain the members' and directors' perspectives. Results from these surveys will inform the strengths and limitations of virtual Clubhouses and provide direction for continued or future endeavors. While our group previously found that virtual Clubhouse engagement was associated with both greater self-reported physical health and lower likelihood of hospitalization in the initial week of the pandemic [8], the current investigation involves a repeated measures approach in which participants were followed for several weeks to gain insight into changes in their physical and mental health over time.

\section{Study Objectives and Research Questions}

The current study's objective is to assess the impact of Clubhouses' efforts in maintaining essential communities virtually for their members in response to the COVID-19 pandemic. Specifically, the aim of this paper includes examination of virtual Clubhouse engagement (high vs. low) and any association to well-being measures (e.g., physical health and mental health). The following hypotheses were addressed: (1) members in the high Clubhouse engagement group will report significantly higher physical and mental health ratings over time than those in the low Clubhouse engagement group, and (2) members who reported contacting peers/members during building closures will report higher physical and mental health ratings over time than those who did not contact other peers/members.

\section{Method}

This study utilized a cross-section of the population of Clubhouse members and performed a secondary analysis of survey data collected during the COVID19 pandemic period of Spring 2020. To assess the well-being of members within their programs, The Clubhouse Member Survey had been administered by Clubhouse International, the Clubhouse accreditation body, and an advisory committee of Clubhouse practitioners, members, and research consultants in the beginning of the COVID-19 pandemic. 
Recruitment for their data collection was conducted by the Clubhouse organizations for the purpose of understanding the impact and response to the pandemic. Survey questions pertained to member coping mechanisms, Clubhouse closures, virtual member engagement, and physical and mental well-being. For full details regarding the survey procedures conducted by Clubhouse International, see Michon et al. [8]. For the current study, secondary data analysis was conducted by the university research team with de-identified data, using the Clubhouse Member Survey sourced from Clubhouse International.

Timeline

During Clubhouse International's data collection for the survey used in this analysis, members were asked to complete a weekly online survey administered by a Clubhouse staff member via a phone call or online conference to gather information on their psychosocial well-being. Given that the survey was voluntary, the number of members participating decreased from Time 1, for which data collection occurred between April 17th and May 11th, 2020, to Time 3, which served as a two-week follow-up to each participant's initial survey (data collection ceased on May 25th, 2020). Due to the fact that fewer members responded to the survey each week, a listwise analysis was conducted for the current study.

\section{Procedure}

Secondary data analysis was conducted using data from The Clubhouse Member Survey. This survey was originally developed in Qualtrics and administered online. The survey was emailed by the accreditation body of Clubhouses (Clubhouse International) on a weekly basis, as an internal assessment of member well-being. An invitation to participate in the surveys was emailed to all 309 Clubhouses with memberships to Clubhouse International. Out of those, 76 (24.6\%) Clubhouses agreed to participate and completed member surveys while 233 (75.4\%) either did not wish to participate or did not respond. A total of 19 out of the 34 countries in which Clubhouses operate [6] participated in surveys, while the majority of responses came from the United States (74.3\%). All Clubhouse members' responses were de-identified, and participants received a member ID number to allow for repeated measurements during data collection. Member consent across survey administrations was obtained through reading or being read an introduction to the survey containing information about consent and the data usage, after which the participant or interviewer proceeded with the first question as indication of consent. For the current study, Institutional Review Board (IRB) exempt human subject's approval was granted through the partner university (IRB-20-04-2040) to assist with secondary data analysis of the survey.

\section{Participants}

The dataset obtained from the first administration of the Clubhouse Member Survey included a total of 1869 participants. During the second and third administrations, 777 and 479 participants responded, respectively. During data cleaning, listwise deletion was used to delete cases with missing data on analysis variables for any of the three time points, leaving a final sample size of 289. This ensured that the current study's analysis included only participants who responded to surveys at all time points. Considering the final total sample size $(n=289)$, slightly more than half of the participants were male $(55 \%)$, as opposed to female $(45 \%)$. The average age of the participants was 46.3 with a range of 20-79 years. Approximately half of participants identified as White/Caucasian (55\%) and nearly a third identified as Black/African American (30\%). The remainder of the participants reported their race/ethnicity as Hispanic $(3 \%)$, American Indian/Alaska Native $(0.3 \%)$, Asian (2\%), or Other/Unknown (9\%).

\section{Measures}

\section{Member Survey}

The Clubhouse Member Survey was designed by an advisory committee comprised of Clubhouse experts and questions were adapted from the Clubhouse Profile Questionnaire (CPQ; [5]). The survey was divided into sections measuring the following: (1) members characteristics, (2) health status, (3) hospitalization visits for medical or psychiatric reasons, (4) employment status, (5) crisis intervention, (6) communication with other Clubhouse members, (7) 
preferred modality of communication, and (8) frequency of virtual engagement with Clubhouses (e.g., daily, weekly, etc.). Additionally, health questions derived from the Clubhouse Profile Questionnaire (CPQ; [5]) assessed the overall self-reported mental and physical health of members prior to and during the COVID-19 pandemic. These questions were assessed across all three time points. Much of this information was collected by Clubhouse International to guide Clubhouses in their response to the COVID-19 pandemic. Select variables were pulled from the dataset for use in the current study to address the research questions.

Clubhouse Engagement/Contact Survey participants were asked to assess their level of contact with Clubhouses. One item prompted respondents to report their frequency of Clubhouse "virtual" engagement (Daily, Weekly, Monthly, or Less Than Monthly). Virtual engagement was defined as meeting via an online or phone modality to connect with Clubhouse peers and staff for programmatic meetings. Participants were grouped during data analysis into a high engagement group (daily or weekly) and a low engagement group (monthly or less than monthly). Participants were also asked to report whether or not they call other members on the phone (yes or no).

Member Well-Being To measure well-being over time, the survey asked participants to rate their mental and physical health separately on scales from 1 to 10 , both currently (at the time of each survey completion) and prior to COVID-19.

\section{Analysis Plan}

Prior to hypothesis testing, preliminary analyses were conducted. To ensure that the main analysis included only participants who responded to all three survey time points, a listwise deletion was performed on missing data. On this final sample, preliminary data analyses were performed to assess for potential confounding variables that must be controlled.

Clubhouse members were then grouped based on the independent variables of interest: (1) high Clubhouse engagement vs. low Clubhouse engagement, and (2) contact with peers/other members vs. no contact with peers/other members. The dependent variables for the main analysis were defined as: (1) self-reported physical health, and (2) self-reported mental health.

To test hypotheses 1 and 2 within one analysis and reduce the likelihood of Type 1 error, a repeated measures multivariate analysis of covariance (MANCOVA) was conducted using the defined dependent and independent variables, while controlling for possible confounding variables. Post-hoc analyses were then conducted to specify which dependent variables (i.e., physical health rating and mental health rating) were significantly impacted by the independent variables of interest.

\section{Results}

Analyses were conducted using IBM SPSS 26 statistical software and secondary data from the Clubhouse Member Survey at all three time points. Descriptive statistics were determined for the sample of participants detailed above $(n=289)$ and are presented in Table 1, and descriptive statistics for participants' selfreported health ratings are presented in Table 2. We arrived at a listwise solution to preserve a consistent sample across time. The original sample included a different number of responses at each wave, beginning with the largest return at Wave $1(1,1869)$, with diminishing completion rates from wave 2 and 3 respectively (Wave $2=777$; Wave $3=479$ ). Therefore, a listwise solution offered the most robust and reliable approach to examine change over time and cases with missing data on the variables of study were excluded. Given the urgency of the study at the time of the pandemic, this approach seemed most viable to gathering a 'pulse' of how members were faring.

Preliminary Analyses and Study Hypotheses

T-tests were conducted to examine whether participants' physical and mental health ratings differed significantly based on member characteristics such as gender and race/ethnicity. There were no significant differences in self-reported prior and current mental health and physical health by country of origin and self- race/ethnicity. There were also no significant differences in physical health by gender. However, mental health rankings were lower among members identifying as female $(M=6.87, S D=2.00)$ as 
Table 1 Final descriptive characteristics for COVID-19 Member Survey sample

\begin{tabular}{|c|c|c|c|}
\hline Variable & $\begin{array}{l}\text { Mean/ } \\
\text { proportion }\end{array}$ & SD & Range \\
\hline $\begin{array}{l}\text { Member's Clubhouse is } \\
\text { accredited }\end{array}$ & 0.88 & & \\
\hline \multicolumn{4}{|l|}{ Member demographics } \\
\hline Age & 46.34 & 13.65 & 59 \\
\hline$\%$ Male & 0.55 & & \\
\hline$\%$ Female & 0.45 & & \\
\hline \multicolumn{4}{|l|}{ Race/ethnicity } \\
\hline White/Caucasian & 0.55 & & \\
\hline Hispanic & 0.03 & & \\
\hline Black/African American & 0.30 & & \\
\hline $\begin{array}{l}\text { American Indian/Alaska } \\
\text { Native }\end{array}$ & 0.003 & & \\
\hline Asian & 0.02 & & \\
\hline Other/unknown & 0.09 & & \\
\hline \multicolumn{4}{|l|}{ Living arrangement } \\
\hline$\%$ Independent housing & 0.49 & & \\
\hline$\%$ Lives with family & 0.32 & & \\
\hline$\%$ Group housing & 0.14 & & \\
\hline$\%$ Clubhouse housing & 0.02 & & \\
\hline$\%$ Shelter & 0.02 & & \\
\hline \multicolumn{4}{|l|}{ Clubhouse attendance } \\
\hline Low frequency & 0.22 & & \\
\hline High frequency & 0.78 & & \\
\hline $\begin{array}{l}\text { Members following a daily } \\
\text { routine }\end{array}$ & 0.90 & & \\
\hline Calls other Clubhouse members ${ }^{\mathrm{a}}$ & 0.60 & & \\
\hline $\begin{array}{l}\text { Utilization of contact with } \\
\text { Clubhouse staff or members as } \\
\text { a coping mechanism }{ }^{\mathrm{b}}\end{array}$ & 0.95 & & \\
\hline
\end{tabular}

$n=289$

${ }^{\mathrm{a}}$ Calls Other Clubhouse Members was taken from Wave 1

${ }^{b}$ Utilization of contact with Clubhouse staff and members as a coping mechanism was taken from Wave 1

compared to male $(M=7.57, S D=1.91)$ in Wave 1 $(t(287)=3.03, p=0.003)$. Therefore, gender was added as covariate in the subsequent analysis. Pearson correlation indicated no significant associations between age and the outcome variables.

\section{Main Analysis}

A repeated measures multivariate analysis of covariance was performed on the two dependent variables associated with self-reported well-being: current mental health status and current physical health status. Adjustment was made for three covariates: gender, and mental and physical health status prior to the pandemic. Independent variables included degree of Clubhouse engagement (high and low) and member/ peer phone contact (presence and absence). See Table 3 for member's mental and physical well-being scores for the frequency of Clubhouse engagement and calling other Clubhouse members across all three time points. The significance value of Box's Test of Equality of Covariance Matrices was larger than $0.001(p=0.002)$, indicating that the analysis did not violate the assumption of homogeneity of variancecovariance matrices. Additionally, all but one of the significance values in Levene's Test of Equality of Error Variances were above 0.05 (current physical health at Wave 3: $p=0.009$ ), indicating equal variances for the majority of variables. In order to account for the violation of assumption, a more conservative alpha level of 0.025 was set for determining significance for current physical health.

With the use of Wilks' criterion, the combined dependent variables were significantly related to the combined covariates, approximate $F(2,281)=28.92$, $p<0.001$, to prior mental health, $F(2,281)=84.01$, $p<0.001$, to prior physical health, with a stronger association between prior and current physical health, $n^{2}=0.37$. There was no significant effect of gender on the combined dependent variables $F(2,281)=0.981$, $p=0.065$. The within subjects MANCOVA with Time as the within-subjects independent variable (Waves 1, 2, 3) was performed on Clubhouse engagement (high and low) and member/peer phone contact (presence and absence). A main effect for Time was significant $F(4,279)=12.4, p<0.001$, along with the interaction between Time and Clubhouse Engagement, $F(4,279)=3.07, p<0.05$ with a small effect size, $n^{2}=0.04$. After adjusting for differences in covariates, the effect of time and maintaining Clubhouse engagement, albeit a small effect size, made a significant contribution to the composite of the dependent variables that best distinguishes between those members who had high engagement (i.e. weekly or daily) as compared to low engagement (i.e. monthly or less than monthly). Member/peer phone contact did not have a significant main effect on the combined dependent variables, nor was there a significant interaction between this variable and Time. 
Table 2 Descriptive statistics for members' health scores

\begin{tabular}{|c|c|c|c|c|c|c|c|c|}
\hline \multirow[t]{2}{*}{ Variable } & \multicolumn{2}{|c|}{ Prior to COVID-19 } & \multicolumn{2}{|c|}{ Time 1} & \multicolumn{2}{|c|}{ Time 2} & \multicolumn{2}{|c|}{ Time 3} \\
\hline & $M$ & $S D$ & $M$ & $S D$ & $M$ & $S D$ & $M$ & $S D$ \\
\hline Physical health & 7.7 & 1.79 & 7.44 & 1.89 & 7.43 & 1.7 & 7.37 & 1.77 \\
\hline Mental health & 7.92 & 1.65 & 7.25 & 1.98 & 7.38 & 1.73 & 7.2 & 1.86 \\
\hline Composite physical + mental health score & 15.62 & 3.08 & 14.69 & 3.49 & 14.81 & 3.12 & 14.58 & 3.35 \\
\hline
\end{tabular}

$n=289$

Table 3 Mental and physical well-being scores for frequency of Clubhouse engagement and calling other Clubhouse members across three time periods.

\begin{tabular}{|c|c|c|c|c|c|c|c|c|c|c|c|c|}
\hline \multirow[t]{2}{*}{ Time period } & \multicolumn{3}{|c|}{ High-frequency engagement } & \multicolumn{3}{|c|}{ Low-frequency engagement } & \multicolumn{3}{|c|}{ Calls other members } & \multicolumn{3}{|c|}{ Does not call other members } \\
\hline & $n$ & $M$ & $S D$ & $n$ & $M$ & $S D$ & $n$ & $M$ & $S D$ & $n$ & $M$ & $S D$ \\
\hline \multicolumn{13}{|l|}{ Mental health } \\
\hline Wave 1 & 224 & 7.29 & 1.96 & 65 & 7.12 & 2.05 & 173 & 7.4 & 1.82 & 116 & 7.03 & 219 \\
\hline Wave 2 & 224 & 7.54 & 1.72 & 65 & 6.86 & 1.66 & 173 & 7.36 & 1.63 & 116 & 7.41 & 1.88 \\
\hline Wave 3 & 224 & 7.37 & 1.83 & 65 & 6.62 & 1.89 & 173 & 7.08 & 1.75 & 116 & 7.4 & 2.02 \\
\hline \multicolumn{13}{|c|}{ Physical health } \\
\hline Wave 1 & 224 & 7.49 & 1.9 & 65 & 7.25 & 1.89 & 173 & 7.54 & 1.82 & 116 & 7.28 & 1.99 \\
\hline Wave 2 & 224 & 7.54 & 1.72 & 65 & 7.03 & 1.61 & 173 & 7.44 & 1.63 & 116 & 7.41 & 1.81 \\
\hline Wave 3 & 224 & 7.54 & 1.73 & 65 & 6.82 & 1.81 & 173 & 7.29 & 1.58 & 116 & 7.5 & 2.01 \\
\hline
\end{tabular}

$n=289$

Post-hoc analyses revealed that statistically significant differences were also present across time, for each dependent variable separately. That is, members who maintained higher Clubhouse engagement over the course of the study period (e.g., 3 weeks), maintained higher self-reported mental and physical health status as compared to low engagement members whose self-reported physical and mental health decreased over time (see Fig. 1).

\section{Discussion}

The purpose of the current study was to examine the extent of member well-being over three time points during the COVID-19 pandemic. The study was designed to take a pulse of how Clubhouses were responding to the pandemic and closures related to social distancing mandates. These changes affected many of the Clubhouses participating in this survey apart from one Clubhouse that remained open but operating under capacity during the study period.
Preliminary descriptive statistics indicated that a majority of participants maintained contact and engagement with their Clubhouse communities during shutdowns. Overall, $60 \%$ of members reported contacting their Clubhouse peers by phone during the study period, and $95 \%$ reported considering contact with Clubhouse members and/or staff a coping mechanism. Further, $78 \%$ of members reported having high Clubhouse engagement, while $22 \%$ reported low Clubhouse engagement. There were no significant differences across countries, indicating that Clubhouse engagement was comparable internationally.

Lastly, a repeated measures MANCOVA was performed to examine the extent of mental or physical health well-being over time. Members who remained highly engaged with their Clubhouse during the study period reported higher wellness rankings over time as compared to those who engaged less frequently, while controlling for gender and prior wellness scores. With the exception of Wave 1, there were no significant differences in well-being between male and female participants. Interestingly, though well-being rankings 
Physical and Mental Health Scores Over Time at High and Low Clubhouse Engagement
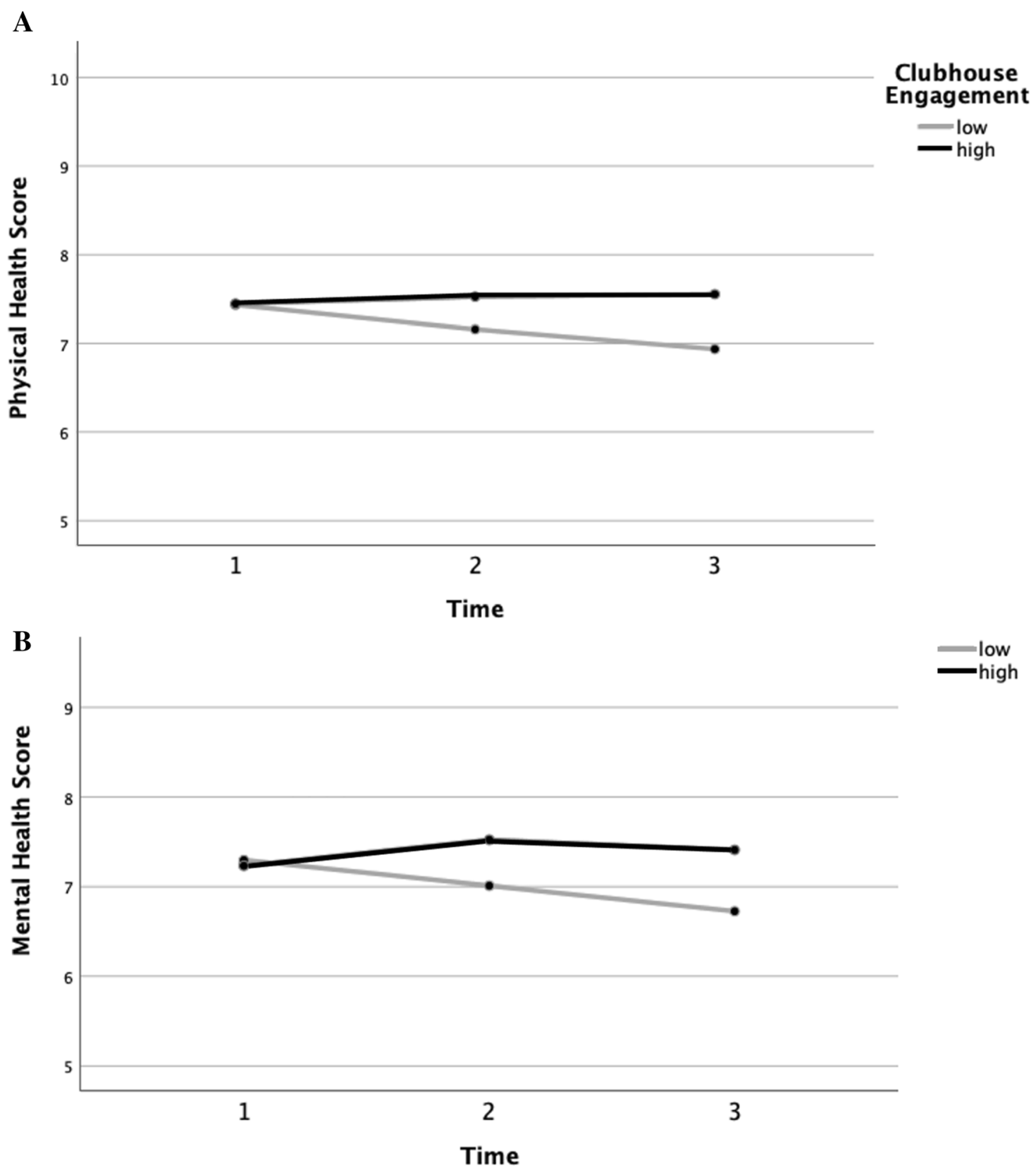

Note. Visual representation of the effect of Clubhouse engagement over time on: A) physical health score, and B) mental health score.

Fig. 1 Physical and mental health scores over time at high and low Clubhouse engagement. Note. Visual representation of the effect of Clubhouse engagement over time on: a physical health score, and b mental health score

were similar for the high engagement group and the low engagement group at Wave 1 , the positive effects of Clubhouse engagement on well-being became visible over time. This suggests that high Clubhouse engagement may have served as a protective factor for members facing the ongoing challenges of the early weeks of the pandemic. Overall, these results may indicate that staying connected with a source of 
support, primarily the Clubhouse community, during a difficult and confusing time was associated with maintaining members' perceived well-being and connection. This finding is essential in understanding how Clubhouses-typically in-person programs-were able to organize and respond effectively to keep their members virtually connected and support their wellbeing.

This dynamic is especially important for people living with serious and persistent mental illness, such as schizophrenia, as a significant aspect of the illness is social withdrawal and isolation $[4,9,11]$. Thus, the Clubhouse community is perceived as an essential factor in members' abilities to cope with the COVID19 pandemic. These findings offer preliminary support for the continuation of virtual Clubhouse conduct programming as a means of supporting member wellbeing, as an alternative or addition to in-person services.

\section{Study Limitations}

An inherent limitation of the study is based on selfreport and the timeliness of the data collected during a significant crisis in our collective history. Due to the voluntary nature of participation and attrition rates across time, the possibility of selection bias limits the generalizability of results. There were, however, no significant differences across analysis variables between those who participated in all three surveys and those who ceased participation, thus mitigating this limitation. Still, our findings regarding the positive effects of virtual Clubhouse services on wellbeing are preliminary; randomized controlled trials are necessary to confirm these correlational results. Further, the study did not examine results by country of origin; given that $75 \%$ of Clubhouses that responded were in the United States, a cross-country comparison of outcomes was outside of the scope of the current investigation. Therefore, the results may be limited in generalizability across countries of origin. Additionally, this study did not examine other organization characteristics, such as specific phases of local quarantines (e.g., building closed or limited access). This may have affected Clubhouse engagement as many Clubhouses were closed while others were at various phases of operating. All Clubhouses involved in this study met the organizational expectation of Clubhouse International, however, which provides some basis for comparison. Moreover, participants were specifically asked to report on levels of virtual engagement, which ensures that results are based on the effects of these virtual Clubhouse interactions.

Strengths of this study include the international collaboration and effort toward documenting the Clubhouse response to COVID-19 during a critical point in time. Clubhouse staff were empowered to assist members in completing online surveys or engaging in an interview regarding their experiences and health status. This interaction may also be perceived as a point of intervention and contact, which could have influenced member reports. However, the question investigating Clubhouse engagement focused on involvement or participation in club activities, conversations, and planning either virtually or in person. Therefore, it is unlikely that participants interpreted the engagement question as including the weekly survey process.

\section{Clinical Implications}

Traditionally, Clubhouses operate within physical spaces to cultivate community and foster recovery from SPMI. During the COVID-19 pandemic, Clubhouse communities have proved essential for those seeking connection with supportive peers and social practitioners, as evidenced by the impact of Clubhouse engagement on member well-being. The transition to virtual services allowed Clubhouses to operate as a global community in an unprecedented yet effective manner. However, indications that Clubhouse members may prefer to remain virtual once the U.S. and countries begin re-opening is yet to be demonstrated.

The results of this study also reveal future implications for reaching a broader population of people recovering from SPMI. Expanding the virtual modality may allow individuals who are not able to engage in Clubhouses in person to still benefit from their services. Future studies during time periods without the pandemic will be necessary to confirm the benefits of the virtual Clubhouse.

\section{Future Directions}

Future research into virtual Clubhouse services may control for the limitations of this study by closely monitoring the level of weekly and daily contact and the types of interactions involved (e.g., adding or 
refining survey items), conducting randomized controlled trials, and involving independent researchers in the data collection processes. Additionally, studies that conduct comparisons to similar programs that only contact their members via email or online communications will be necessary to assess whether virtual Clubhouses are comparable to typical online health-related services.

\section{Conclusion}

These findings provide preliminary evidence that virtual Clubhouse services may represent an effective means of supporting member well-being, though future research is necessary to confirm these findings. The study highlights the effectiveness of Clubhouses' adaptations to the pandemic and indicates that quickly shifting to a virtual platform was likely successful in keeping some of the most vulnerable consumers connected to mental health and community during the pandemic. Further, this study demonstrates the possibility for alternative methodology to keeping people connected and an innovation towards the use of virtual Clubhouses. Future investigation into these virtual services may further elucidate these associations, as Clubhouses reinvent methods of engaging members both within and outside the Clubhouse walls-remaining an essential and vital service to people who are living within the margins of our society.

\section{Declarations}

Conflict of interest On behalf of all authors, the corresponding author states that there is no conflict of interest.

Funding No funding.

\section{References}

1. Adlhoch C, Baka A, Ciotti M, Dias JG, Kinsman J, Leitmeyer K, et al. Considerations relating to social distancing measures in response to COVID-19-second update. European Centre for Disease Prevention and Control Report. 2020.

2. Bettinsoli ML, Napier JL, Di Riso D, Moretti L, Delmedico M, Piazzolla A, Moretti B, Bettinsoli P. Mental health conditions of Italian healthcare professionals during the COVID-19 disease outbreak. Appl Psychol Health WellBeing. 2020. https://doi.org/10.1111/aphw.12239.
3. Carolan M, Onaga E, Pernice-Duca F, Jimenez T. A place to be: the role of clubhouses in facilitating social support. Psychiatr Rehabil J. 2011;35(2):125-32. https://doi.org/10. 2975/35.2.2011.125.132.

4. Chang CW, Chung CL, Biegel DE, Pernice-Duca F, Min MO, D'Angelo L. Predictors of loneliness of clubhouse members. Psychiatr Rehabil J. 2014;37(1):51-4. https://doi. org/10.1037/prj0000052.

5. Clubhouse International. Clubhouse Profile Questionnaire. 2012. http://clubhouse-intl.org/images/download_discuss_ cpq.pdf.

6. Clubhouse International. Clubhouse Directory. 2021. https://clubhouse-intl.org/what-we-do/internationaldirectory/.

7. McKay C, Nugent KL, Johnsen M, Eaton WW, Lidz CW. A systematic review of evidence for the clubhouse model of psychosocial rehabilitation. Adm Policy Ment Health Ment Health Serv Res. 2018;45(1):28-47. https://doi.org/10. 1007/s10488-016-0760-3.

8. Michon A, Hinchey LM, Pernice F, Drews J, Price M, Christian J, Rice K, Kellogg L. Responding to the global pandemic: a pulse of the well-being of clubhouse communities moving virtual. J Psychosoc Rehabil Ment Health. 2021. https://doi.org/10.1007/s40737-021-00217-9.

9. Perese EF, Wolf M. Combating loneliness among persons with severe mental illness: social network interventions' characteristics, effectiveness, and applicability. Issues Ment Health Nurs. 2005;26(6):591-609. https://doi.org/10.1080/ 01612840590959425.

10. Pietrabissa G, Simpson SG. Psychological consequences of social isolation during COVID-19 outbreak. Front Psychol. 2020;11:1-4. https://doi.org/10.3389/fpsyg.2020.02201.

11. Prince JD, Oyo A, Mora O, Wyka K, Schonebaum AD. Loneliness among persons with severe mental illness. J Nerv Ment Dis. 2018;206(2):136-41. https://doi.org/10. 1097/NMD.0000000000000768.

12. Rajkumar RP. COVID-19 and mental health: a review of the existing literature. Asian J Psychiatr. 2020;52:1-5. https:// doi.org/10.1016/j.ajp.2020.102066.

13. Rothan HA, Byrareddy SN. The epidemiology and pathogenesis of coronavirus disease (COVID-19) outbreak. J Autoimmun. 2020;109:1-4. https://doi.org/10.1016/j.jaut. 2020.102433.

14. The Lancet. India under COVID-19 lockdown. Lancet (London, England). 2020;395(10233):1315. doi:https://doi. org/10.1016/S0140-6736(20)30938-7.

15. Usher K, Durkin J, Bhullar N. The COVID-19 pandemic and mental health impacts. Int $\mathrm{J}$ Ment Health Nurs. 2020;29(3):315-8. https://doi.org/10.1111/inm.12726.

16. Vindegaard N, Benros ME. COVID-19 pandemic and mental health consequences: Systematic review of the current evidence. Brain Behav Immun. 2020;89:531. https:// doi.org/10.1016/j.bbi.2020.05.048.

Publisher's Note Springer Nature remains neutral with regard to jurisdictional claims in published maps and institutional affiliations. 